\title{
Variational multi-scale approximation of the one-dimensional forced Burgers equation: the role of orthogonal sub-grid scales in turbulence modeling
}

\author{
Camilo Bayona, Joan Baiges* and Ramon Codina \\ Centre Internacional de Mètodes Numèrics en Enginyeria (CIMNE), Edifici C1, Campus Nord UPC C/Gran Capità S/N \\ 08034 Barcelona, Spain \\ Universitat Politècnica de Calatunya, Jordi Girona 1-3, Edifici C1, 08034 Barcelona, Spain
}

\begin{abstract}
SUMMARY
A numerical approximation for the one-dimensional Burgers equation is proposed by means of the Orthogonal Sub-Grid Scales - Variational Multi-Scale (OSGS-VMS) method. We evaluate the role of the variational subscales in describing the Burgers "turbulence" phenomena. Particularly, we seek to clarify the interaction between the subscales and the resolved scales when the former are defined to be orthogonal to the finite dimensional space. Direct Numerical Simulation (DNS) is used to evaluate the resulting OSGS-VMS energy spectra. The comparison against a Large Eddy Simulation (LES) model is presented for numerical discretizations in which the grid is not capable of solving the small scales. An accurate approximation to the phenomena of turbulence is obtained with the addition of the purely dissipative numerical terms given by the OSGS-VMS method without any modification of the continuous problem. Copyright (c) 0000 John Wiley \& Sons, Ltd.
\end{abstract}

Received ...

KEY WORDS: Burgers equation; Turbulence; Variational multi-scale method; Orthogonal sub-grid scales; Large eddy simulation; Direct numerical simulation

\section{INTRODUCTION}

The Burgers equation is a simplified model of the equations that govern fluid flow, and in spite of its simplicity it shares some features of the nonlinear dynamics present in the Navier-Stokes equations. Burgers attempted unsuccessfully to arrive at a statistical theory of turbulent fluid motion in [2], but he was able to clarify the interaction between transient, dissipative, and nonlinear terms in an extremely simplified equation of motion. His contribution still represents a significant achievement when one aims to describe turbulence: Burgers turbulence exhibits an energy cascade that is similar to that encountered in fluid flow turbulence governed by the Navier-Stokes equations, containing also an inertial and a dissipation range.

Analytic solutions are known for the forced Burgers equation: when the nonlinear term is dominant it leads to the formation of shocks, and the solution exhibits an energy spectrum that behaves as $k^{-2}$ in the inertial range, $k$ being the wavenumber. A complete review of the analytical solutions by Hopf [18] and Cole [9], including some of the mathematical and computational framework for the viscous form of the time-dependent Burgers equation, has been reported in [27]. Nevertheless, the important difference between the Navier-Stokes and the Burgers dynamics is that the smallest scales that dissipate the energy of the flow in the Burgers equation do not refer to the smallest eddies, but to the shock thickness instead.

$\overline{{ }^{*} \text { Correspondence }}$ to: jbaiges@ cimne.upc.edu

Copyright (C) 0000 John Wiley \& Sons, Ltd.

Prepared using fldauth.cls [Version: 2010/05/13 v2.00] 
Numerically resolving every scale of the solution is what is referred as the Direct Numerical Simulation (DNS). Most of the numerical approaches that aim to correctly describe turbulent flows can be prohibitively expensive for nearly all systems with complex geometries, since the discrete meshes of the approximation (or the number of basis functions in the approximation space) need to be refined up to a power of the Reynolds number. Hence, it is presently impossible to simulate flows at high Reynolds numbers using DNS, and in most of the practical fluid dynamics applications turbulence phenomena are modeled somehow.

Several alternatives have raised in the turbulence modeling community, being Large Eddy Simulation (LES) one of the most robust and prevailing approaches. The basic idea of this method is to filter the fluid equations in space and time, simulating only the filtered large scales, while the smallest (and most expensive to compute) scales are incorporated into the overall solution by including a modelling term in the filtered equations. The filtered scales are called the resolved scales of the flow, the scales below the resolved scales are called the subscales. However, filtering the equations results in an unclosed term involving the subscales that cannot be determined from the resolved quantities, so that it needs to be calculated by assuming some a priori properties of the flow. In particular, eddy viscosity type models, such as the Smagorinsky model [26] and the Germano model [13], apply proportionality constants that must be set empirically a priori, and this makes them incomplete models. Some other authors, such as [24, 14], approximated the problem by decomposing the resolved and unresolved scales into deterministic and stochastic components, in which the subscales are calculated by using a stochastic estimation. And yet, as reviewed by [11], all these models fail to accurately represent inhomogeneous flows.

Another method that separates the solution into resolved and unresolved scales is the Variational Multi-Scale (VMS) method [19]. Alternatively to the LES approach, this method splits the solution in order to stabilize the numerical approximation of the problem. Fluid flow equations have been traditionally stabilized by using the family of VMS methods, including the Algebraic Sub-Grid Scales (ASGS) and the Orthogonal Sub-Grid Scales (OSGS) methods (like in [4]). The original idea of using the multiscale formulation with local approximation to the fine scales to compute turbulent flows was introduced in [6], and later discussed in [20]. The illustration that the VMS formulation works as a turbulent model, that depends on the validity of the approximation made to derive the evolution equation for the unresolved scales, was elaborated in $[8,16]$. Some other authors proposed to further split the resolved scales into coarse and fine scales, and to adopt the hypothesis that the subscales do not affect the coarse resolved scales. Among these methods we can distinguish $[29,15]$, in which the effect of the unresolved scales is introduced only into the fine resolved scales. More recently, [17, 1, 23, 10, 12] have directly applied the VMS method in order to solve the Navier-Stokes turbulence, in what are called Implicit LES (ILES) techniques. This approach accurately solves turbulence relying on the addition of dissipative numerical terms solely, and without any modification of the continuous problem.

In this work we aim to apply the OSGS-VMS method to the numerical approximation of the one-dimensional forced Burgers equation. In the spirit of ILES methods, we aim to clarify the mechanisms through which the resolved and unresolved scales interact with each other, and remark about the role of the orthogonal sub-grid scales in modeling Burgers turbulence. The proposed numerical approximation exploits the Fourier transform of the Burgers equation, following the work in [28], in which the scale dependence on the numerical dissipation introduced by the subscales is clarified. Complementary to that approach, we include the orthogonal sub-grid scales into the resolved scales equation by means of the adjoint of the nonlinear operator applied to the test function, so that both terms associated with the Cross and Reynolds stresses are accounted for. Solving the subscales in a separated equation (subscales equation) leads to the inclusion of a Leonard stress term. In addition, we propose to calculate subscales in terms of the resolved scales by defining a priori the space where the subscales exist (as the orthogonal space to the finite dimensional resolved space), and approximating the nonlinear operator associated to the Burgers problem. The scale dependence of the introduced numerical dissipation terms is studied by considering the triadic interactions among the resolved scales and the subscales. More precisely, terms associated with the Leonard, Cross and Reynolds stresses (involving resolved and unresolved 
scales) allow forward and backward scattering. Contrary to [22], we are not interested in transient turbulent solutions. Instead, we test the OSGS-VMS method in contrast to the static Smagorinksy LES model, and present some conclusions about the behavior of the orthogonal subscales in the numerical approximation of the Burgers turbulence phenomena.

The outline of this article is organized as follows. In Section 2 we define the Burgers equation and the VMS form of the problem. The numerical approximation used to solve the problem is presented in Section 3. Section 4 shows the numerical results for a test example. OSGS-VMS results are compared both to DNS and LES simulations at the end of that section. Finally, conclusions are stated in Section 5.

\section{PROBLEM DEFINITION}

In this section we define the VMS approximation of the Burgers problem. First, we recall the onedimensional forced Burgers equation in the physical space, and derive the Galerkin form of the problem. Then, we apply the VMS framework and present the equations for both the resolved scales and the subscales.

\subsection{Burgers equation}

The one-dimensional forced Burgers equation [3] consists of finding a scalar function $u(x, t)$ of position $x \in \Omega=(0,2 \pi)$ and of time $t \geq 0$ such that, given a forcing term $f(x, t)$,

$$
\partial_{t} u+u \partial_{x} u-v \partial_{x x} u=f, \quad x \in(0,2 \pi), t>0,
$$

with an initial condition $u(x, 0)=u^{0}(x)$ in $\Omega$ and periodic boundary conditions at $x=0$ and $x=2 \pi$, $t \geq 0$. The diffusivity coefficient $v$ is considered as homogeneous and constant.

The Burgers equation shares some of the properties of the Navier-Stokes equations. Apart from the temporal derivative, the left-hand-side (LHS) contains both a nonlinear and a linear term, associated to convection and diffusion, respectively. The interaction between the dissipation given by the diffusive term (containing second order derivatives) and the convection given by the nonlinear inertial term also appears in the incompressible Navier-Stokes equations.

For convenience Eq. (1) can be written in the form

$$
\partial_{t} u+\mathscr{L}(u ; u)=f, \quad x \in(0,2 \pi), t>0,
$$

by introducing the bilinear operator $\mathscr{L}(u ; v)=u \partial_{x} v-v \partial_{x x} v$. For smooth solutions, $\mathscr{L}(u ; u)=$ $u \partial_{x} u-v \partial_{x x} u=\partial_{x}\left(\frac{1}{2} u^{2}-v \partial_{x} u\right)$. The latter is the form that admits physically meaningful solutions when they develop discontinuities; it is known as the conservation form.

Let $\mathscr{W}=H_{\text {per }}^{1}(\Omega)$ be the subspace of periodic functions in $H^{1}(\Omega)$, and let us write $(f, g)=\int_{\Omega} f g$ for any two functions $f$ and $g$. Introducing the form

$$
A(u ; w, v):=-\frac{1}{2}\left(u \partial_{x} w, v\right)+v\left(\partial_{x} w, \partial_{x} v\right),
$$

the variational form of the problem can be written as: find $u: \mathbb{R}^{+} \longrightarrow \mathscr{W}$ such that

$$
\begin{aligned}
\left(w, \partial_{t} u\right)+A(u ; w, u) & =(w, f), & t>0, \\
(w, u) & =\left(w, u^{0}\right), & t=0,
\end{aligned}
$$

for all $w \in \mathscr{W}$

\subsection{Variational multi-scale method}

Let us consider a finite-dimensional subspace $\mathscr{W}_{N} \subset \mathscr{W}$, of dimension $N$, which approximates $\mathscr{W}$ as $N \rightarrow \infty$. The Galerkin approximation to problem (4)-(5) can be stated as follows: find 
$u_{N}: \mathbb{R}^{+} \longrightarrow \mathscr{W}_{N}$ such that

$$
\begin{aligned}
\left(w_{N}, \partial_{t} u_{N}\right)+A\left(u_{N} ; w_{N}, u_{N}\right) & =\left(w_{N}, f\right), \quad t>0, \\
\left(w_{N}, u_{N}\right) & =\left(w_{N}, u^{0}\right), \quad t=0,
\end{aligned}
$$

for all $w_{N} \in \mathscr{W}_{N}$. This approximation suffers from instability problems, which vary according to the way to construct $\mathscr{W}_{N}$, but which are in any case due to the convective property of the nonlinear term.

The idea of the VMS method is to decompose the space of the unknown into a finite-dimensional space $\mathscr{W}_{N}$, and an infinite-dimensional one, $\tilde{\mathscr{W}}$, so that $\mathscr{W}=\mathscr{W}_{N} \oplus \tilde{\mathscr{W}}$. The unknown and the test functions are accordingly split as $u=u_{N}+\tilde{u}$ and $w=w_{N}+\tilde{w}$, respectively. We shall refer to functions in $\mathscr{W}_{N}$ as the large or resolved scales and to functions in $\tilde{\mathscr{W}}$ as the subscales or unresolved scales.

Equation (4) can be equivalently written as the system of equations

$$
\begin{array}{cc}
\left(w_{N}, \partial_{t} u\right)+A\left(u ; w_{N}, u\right)=\left(w_{N}, f\right), & \text { for all } w_{N} \in \mathscr{W}_{N}, t>0 \\
\left(\tilde{w}, \partial_{t} u\right)+A(u ; \tilde{w}, u) & =(\tilde{w}, f), \quad \text { for all } \tilde{w} \in \tilde{W}, t>0
\end{array}
$$

and likewise for the initial condition, Eq. (5). The second term in the LHS of Eq. (8) can be split as

$$
A\left(u ; w_{N}, u\right)=A\left(u_{N} ; w_{N}, u_{N}\right)+A\left(u_{N} ; w_{N}, \tilde{u}\right)+A\left(\tilde{u} ; w_{N}, u_{N}\right)+A\left(\tilde{u} ; w_{N}, \tilde{u}\right)
$$

We may define:

$$
\begin{array}{ll}
A\left(u_{N} ; w_{N}, u_{N}\right) & \text { Galerkin terms } \\
A\left(u_{N} ; w_{N}, \tilde{u}\right)+A\left(\tilde{u} ; w_{N}, u_{N}\right) & \text { Nonlinear Cross terms } \\
A\left(\tilde{u} ; w_{N}, \tilde{u}\right) & \text { Nonlinear contribution of the unresolved scales }
\end{array}
$$

If $\mathscr{W}_{N}$ is made of smooth functions, as it is the case considered below, it is readily seen that the equation for the unresolved scales (9) can be written as

$$
\left(\tilde{w}, \partial_{t} \tilde{u}\right)+(\tilde{w}, \mathscr{L}(u ; \tilde{u}))=\left(\tilde{w}, f-\partial_{t} u_{N}-\mathscr{L}\left(u ; u_{N}\right)\right), \quad \text { for all } \tilde{w} \in \tilde{\mathscr{W}}, t>0
$$

The objective of the VMS method is to approximate the subscales in terms of the resolved scales, in order to end up with a problem for the resolved scales alone. The key point is the approximation of $\mathscr{L}(u ; \tilde{u})$ in order to make Eq. (14) easily solvable. We will not describe the motivation, which can be based on a Fourier analysis of the problem, but the approximation that we consider is (see [5])

$$
\mathscr{L}(u ; \tilde{u}) \approx \tau^{-1}(u) \tilde{u},
$$

where $\tau(u)>0$ is a numerical parameter of the formulation, the expression of which is given later for a particular approximation. The objective of (15) is not to provide an accurate pointwise approximation to $\tilde{u}$, but to capture its effect on the resolved scales.

The residual of the resolved scales is defined as $R_{N}\left(u ; u_{N}\right)=f-\partial_{t} u_{N}-\mathscr{L}\left(u ; u_{N}\right)$. If approximation (15) is inserted in (14), one obtains a nonlinear problem for $\tilde{u}$. Even though it is possible to deal with this nonlinearity (see [5]), we will consider that the unresolved scales are smaller than the resolved ones, so that

$$
\tau(u) \approx \tau\left(u_{N}\right), \quad R_{N}\left(u ; u_{N}\right) \approx R_{N}\left(u_{N} ; u_{N}\right)
$$

These approximations and (15) allow us to write the approximate equation for the subscales as

$$
\left(\tilde{w}, \partial_{t} \tilde{u}+\tau^{-1}\left(u_{N}\right) \tilde{u}\right)=\left(\tilde{w}, R_{N}\left(u_{N} ; u_{N}\right)\right), \quad \text { for all } \tilde{w} \in \tilde{\mathscr{W}}, t>0
$$

Even though we have not distinguished them, the subscales solution of (17) are approximate, whereas those solution of (14) are exact. 
The subscales space is generally defined in two different ways. The first, and the most common approach, is to define it as the space of residuals of the resolved scales, and therefore to consider that

$$
\partial_{t} \tilde{u}+\tau^{-1}\left(u_{N}\right) \tilde{u}=R_{N}\left(u_{N} ; u_{N}\right), t>0 .
$$

This is what we call Algebraic Sub-Grid Scales (ASGS) method in finite elements. The second approach is the Orthogonal Sub-Grid Scales (OSGS) method, which defines the subscales space as the space orthogonal to the resolved scales space. If $P_{N}$ is the $L^{2}$-projection onto $\mathscr{W}_{N}$ and $P_{N}^{\perp}=I-P_{N}, I$ being the identity, then the equation for the unresolved scales in this case is

$$
\partial_{t} \tilde{u}+\tau^{-1}\left(u_{N}\right) \tilde{u}=P_{N}^{\perp}\left[R_{N}\left(u_{N} ; u_{N}\right)\right], t>0 .
$$

In the spectral approximation described next, we shall see that the OSGS method is in fact the natural approach, since the basis functions are mutually $L^{2}$-orthogonal (and also $H^{1}$-orthogonal). In this case, we thus have that $\mathscr{W}=\mathscr{W}_{N} \oplus \mathscr{W}_{N}^{\perp}$, with $\mathscr{W}_{N}^{\perp}=\tilde{\mathscr{W}}$.

\section{SOLUTION METHOD}

In the previous section, we have defined the variational formulation for each scale of the Burgers problem. The two sub-problems (8) and (17) are now numerically approximated by transforming them into the Fourier space or, equivalently, by using a spectral Fourier method. Considering $\tilde{u}=0$ would reduce the problem to be solved to (6)-(7), i.e., the Galerkin-Fourier method. It is well known that this approximation of the Burgers equation suffers from numerical instabilities. In this section we recall the importance of the numerical diffusion introduced by the VMS formulation, not only to prevent spurious oscillations in the numerical solution, but also to model the "turbulence" phenomena. Next, we derive the energy equation in Fourier space. Finally, the time integration scheme is described.

\subsection{Discrete Burgers equation}

Let us consider $N$ even and define the discrete space where the solution is sought as

$$
\mathscr{W}_{N}:=\operatorname{span}\left\{\mathrm{e}^{\mathrm{i} k x}: k \in[-N / 2, N / 2-1] \subset \mathbb{N}\right\},
$$

i.e., the space spanned by $N$ Fourier modes, $k$ being the wavenumber. Let us write $(f, g)=\int_{\Omega} \bar{f} g$ for the $L_{2}$ inner product over complex-valued functions $f$ and $g$, by denoting the complex conjugate with an overline. The approximate unknown can thus be expressed as

$$
u_{N}(x, t)=\sum_{k=-N / 2}^{N / 2-1} \hat{u}_{k}(t) \mathrm{e}^{\mathrm{i} k x}
$$

where $\hat{u}_{k}(t):=\left(u_{N}(x, t), \mathrm{e}^{\mathrm{i} k x}\right)$. We recall the orthogonality relation $\left(\mathrm{e}^{\mathrm{i} k x}, \mathrm{e}^{\mathrm{i} l x}\right)=2 \pi \delta_{k l}$, where $\delta_{k l}$ is the Kronecker delta. The notation $\sum_{k=-N / 2}^{N / 2-1} \hat{u}_{k}(t) \mathrm{e}^{\mathrm{i} k x}:=\sum_{k} \hat{u}_{k} \mathrm{e}^{\mathrm{i} k x}$ will be used in the following.

To obtain the discrete weak form of the problem, let us test the differential equation (8) with the basis functions of $\mathscr{W}_{N}$. If $\hat{f}_{l}, l \in \mathbb{N}$, are the Fourier coefficients of the forcing term $f$, we have that $\left(\mathrm{e}^{\mathrm{i} k x}, \sum_{l} \hat{f}_{l} \mathrm{e}^{\mathrm{i} l x}\right)=2 \pi \hat{f}_{k}$. For the transient and diffusive terms we have

$$
\left(\mathrm{e}^{\mathrm{i} k x}, \partial_{t} \sum_{l} \hat{u}_{l} \mathrm{e}^{\mathrm{i} l x}\right)=2 \pi \partial_{t} \hat{u}_{k}, \quad\left(v \partial_{x} \mathrm{e}^{\mathrm{i} k x}, \partial_{x} \sum_{l} \hat{u}_{l} \mathrm{e}^{\mathrm{i} l x}\right)=-2 \pi v k^{2} \hat{u}_{k}
$$

for all $k, l \in[-N / 2, N / 2-1]$ and $t>0$. Moreover, the nonlinear Galerkin term results in

$$
\left(\frac{1}{2} \sum_{q} \hat{u}_{q} \mathrm{e}^{\mathrm{i} q x} \partial_{x} \mathrm{e}^{\mathrm{i} k x}, \sum_{l} \hat{u}_{l} \mathrm{e}^{\mathrm{i} l x}\right)=-\pi \mathrm{i} k \sum_{q+l=k} \hat{u}_{q} \hat{u}_{l},
$$


for all $k, q, l \in[-N / 2, N / 2-1]$ and $t>0$. The remaining terms of Eq. (10), which depend on the subscales, are developed next. Note first that the transient and diffusive terms belong to $\mathscr{W}_{N}$, and we may assume that $f$ also belongs to this space without losing accuracy, since the order of the error made will be the same as that of approximating $\mathscr{W}$ by $\mathscr{W}_{N}$. Therefore, if the subscales are defined to belong to the space orthogonal to the finite-dimensional resolved space, then the subscales equation can be expressed as

$$
\left(\tilde{w}, \partial_{t} \tilde{u}+\tau^{-1}\left(u_{N}\right) \tilde{u}\right)=\left(\tilde{w},-u_{N} \partial_{x} u_{N}+P_{N}\left(u_{N} \partial_{x} u_{N}\right)\right), \quad \text { for all } \tilde{w} \in \tilde{\mathscr{W}} .
$$

Now, the term $u_{N} \partial_{x} u_{N}$ can be developed as

$$
u_{N} \partial_{x} u_{N}=\sum_{q} \mathrm{e}^{\mathrm{i} q x} \hat{u}_{q} \sum_{l} \mathrm{i} l \mathrm{e}^{\mathrm{i} l x} \hat{u}_{l}=\sum_{q, l} \mathrm{i} l \mathrm{e}^{\mathrm{i}(q+l) x} \hat{u}_{q} \hat{u}_{l}
$$

for all $q, l \in[-N / 2, N / 2-1]$. This term belongs to the space $\operatorname{span}\left\{\mathrm{e}^{\mathrm{i} r x}: r \in[-N, N-2]\right\}$. The projection onto the space of resolvable scales, $P_{N}$, can be obtained by considering in the sum above only the wavenumbers $q, l$, that satisfy $q+l \in[-N / 2, N / 2-1]$. Consequently, the orthogonal subscales belong to the Fourier space $\tilde{\mathscr{W}}$, given by

$$
\tilde{\mathscr{W}}:=\operatorname{span}\left\{\mathrm{e}^{\mathrm{i} r x}: r \in[-N, N-2] \backslash[-N / 2, N / 2-1]\right\} .
$$

With this definition, taking $\tilde{w}=\mathrm{e}^{\mathrm{i} r x}$ in (19), we obtain the equation for the subscales

$$
\partial_{t} \hat{\tilde{u}}_{r}+\tau^{-1}\left(u_{N}\right) \hat{\tilde{u}}_{r}=\sum_{q+l=r} \mathrm{i} l \hat{u}_{q} \hat{u}_{l},
$$

for all $q, l \in[-N / 2, N / 2-1], r \in[-N, N-2] \backslash[-N / 2, N / 2-1]$, and $t>0$.

In order to close the subscales calculation, the approximation of the Burgers nonlinear operator (15) is needed. The key point in the design of the VMS formulation is the construction of $\tau$. In this work we use the approximation of the nonlinear Burgers operator proposed in [28], which is defined as

$$
\tau\left(u_{N}\right)=\left[3 \pi v^{2}\left(\frac{4}{h^{2}}\right)^{2}+\frac{4}{h^{2}}\left\|u_{N}\right\|^{2}\right]^{-\frac{1}{2}},
$$

where $h=\pi / N$ can be considered as an effective grid size and $\left\|u_{N}\right\|$ is the $L^{2}$-norm of $u_{N}$. This approximation basically aims to bound the effect of the nonlinear operator in Fourier space. Now we can compute the subscales in terms of the resolvable scales from (20) and use the resulting expression in the equation for the latter.

\section{Remark 1}

The space of subscales is in principle of infinite dimension, and defined as the complimentary of the Fourier space of resolved scales. It can also be defined as the difference between the DNS solution and the resolved space, such as the one derived in [28]. However, with the approximations we have assumed, the space of subscales turns out to be of finite dimension, and defined in terms of the finitedimensional resolved space. In particular, the Fourier space where the orthogonal subscales live has the same dimension as the resolved space, and a finer discretization generates a richer definition of the subscales.

\section{Remark 2}

We could actually solve the subscales taking into account the nonlinearity of the problem, that is to say, we could proceed without approximation (16). This simply amounts to replace $u$ by $u_{N}+\tilde{u}$ and deal with the additional nonlinearity by using an appropriate linearization scheme.

\section{Remark 3}

Neglecting the time derivative in (20) leads to the so-called quasi-static subscales, which could be understood as the subscales given automatically by the residual. On the contrary, by defining the 
subscales equation as in (20), it must be solved as a separated differential equation of the problem. This corresponds to what was termed as dynamic subscales in [5]. Note that Eq. (20) is in fact an ordinary differential equation that needs to be integrated in time for each Fourier mode.

Let us look now at the contribution of the subscales into the finite-dimensional equation (10). For conciseness, let us imply $q, k \in[-N / 2, N / 2-1], r \in[-N, N-2] \backslash[-N / 2, N / 2-1]$, and $t>0$. Since the subscales are orthogonal to the finite-dimensional space, and the diffusive term in (13) belongs to the resolved space, it reduces to $\left(v \partial_{x x} \mathrm{e}^{\mathrm{i} k x}, \sum_{r} \hat{\tilde{u}}_{r} \mathrm{e}^{\mathrm{i} r x}\right)=0$. The same occurs with the transient term for the subscales (13), $\left(\mathrm{e}^{\mathrm{i} k x}, \partial_{t} \sum_{r} \hat{\tilde{u}}_{r} \mathrm{e}^{\mathrm{i} r x}\right)=0$, which is orthogonal to the resolved scales by definition. Regarding the nonlinear terms that involve the subscales in (10), each one is developed as follows:

$$
\begin{aligned}
& \left(\frac{1}{2} \sum_{q} \hat{u}_{q} \mathrm{e}^{\mathrm{i} q x} \partial_{x} \mathrm{e}^{\mathrm{i} k x}, \sum_{r} \hat{\tilde{u}}_{r} \mathrm{e}^{\mathrm{i} r x}\right)=-\pi \mathrm{i} k \sum_{q+r=k} \hat{u}_{q} \hat{\tilde{u}}_{r}, \\
& \left(\frac{1}{2} \sum_{r} \hat{\tilde{u}}_{r} \mathrm{e}^{\mathrm{i} r x} \partial_{x} \mathrm{e}^{\mathrm{i} k x}, \sum_{q} \hat{u}_{q} \mathrm{e}^{\mathrm{i} q x}\right)=-\pi \mathrm{i} k \sum_{r+q=k} \hat{\tilde{u}}_{r} \hat{u}_{q}, \\
& \left(\frac{1}{2} \sum_{r} \hat{\tilde{u}}_{r} \mathrm{e}^{\mathrm{i} r x} \partial_{x} \mathrm{e}^{\mathrm{i} k x}, \sum_{p} \hat{\tilde{u}}_{p} \mathrm{e}^{\mathrm{i} p x}\right)=-\pi \mathrm{i} k \sum_{r+p=k} \hat{\tilde{u}}_{r} \hat{\tilde{u}}_{p} .
\end{aligned}
$$

As we can solve each Fourier wavenumber $k$ separately, the final expression for the resolvable scales of the Burgers equation is

$$
\partial_{t} \hat{u}_{k}+\frac{\mathrm{i} k}{2} \sum_{q+l=k} \hat{u}_{q} \hat{u}_{l}+\mathrm{i} k \sum_{q+r=k} \hat{u}_{q} \hat{\tilde{u}}_{r}+\frac{\mathrm{i} k}{2} \sum_{r+p=k} \hat{\tilde{u}}_{r} \hat{\tilde{u}}_{p}+v k^{2} \hat{u}_{k}=\hat{f}_{k}
$$

for all $k, q, l \in[-N / 2, N / 2-1], r, p \in[-N, N-2] \backslash[-N / 2, N / 2-1]$, and $t>0$.

The formulation is now complete. It consists of solving (25) together with (20), for $u_{N}$ and $\tilde{u}$.

\section{Remark 4}

The problem has to be completed with initial conditions for (20). We assume that $\hat{\tilde{u}}_{r}^{0}=0$, for all $r \in[-N, N-2] \backslash[-N / 2, N / 2-1]$, which means that we assume for simplicity that the initial condition belongs to the space of resolvable scales.

\subsection{Discrete energy equation}

We now develop the numerical approximation to the energy equation in Fourier space. The energy $e_{k}$ of the $k$-th wavenumber is obtained by taking the product of $\hat{u}_{k}$ with its complex conjugate $\hat{u}_{k}$ (and dividing by 2 , if wished). Its time derivative is given by:

$$
\partial_{t} e_{k}=\partial_{t}\left(\hat{u}_{k} \overline{\hat{u}_{k}}\right)=\hat{u}_{k} \partial_{t} \overline{\hat{u}_{k}}+\overline{\hat{u}_{k}} \partial_{t} \hat{u}_{k} .
$$

After the right-hand-side terms of the previous equation are developed, the discrete energy equation can be written as:

$$
\partial_{t} e_{k}=-v 2 k^{2} e_{k}-T(k)-C(k)-R(k)+\overline{\hat{u}}_{k} \hat{f}_{k}+\overline{\hat{f}}_{k} \hat{u}_{k},
$$

for all $k \in[-N / 2, N / 2-1]$, and $t>0$, where

$$
T(k)=\overline{\hat{u}_{k}}\left(\frac{\mathrm{i} k}{2} \sum_{q+l=k} \hat{u}_{q} \hat{u}_{l}\right)+\hat{u}_{k}\left(\overline{\frac{\mathrm{i} k}{2} \sum_{q+l=k} \hat{u}_{q} \hat{u}_{l}}\right)
$$

stands for the nonlinear transfer term due to the resolved scales,

$$
C(k)=\overline{\hat{u}_{k}} \mathrm{i} k \sum_{q+r=k} \hat{u}_{q} \hat{\tilde{u}}_{r}+\hat{u}_{k} \overline{\mathrm{i} k \sum_{q+r=k} \hat{u}_{q} \hat{\tilde{u}}_{r}}
$$



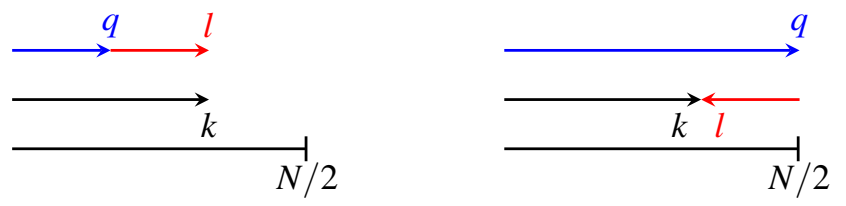

Figure 1. Triadic interactions among the resolved scales

is the transfer due to the Cross nonlinear term involving resolved scales and subscales, and

$$
R(k)=\overline{\hat{u}_{k}}\left(\frac{\mathrm{i} k}{2} \sum_{r+p=k} \hat{\tilde{u}}_{r} \hat{\tilde{u}}_{p}\right)+\hat{u}_{k}\left(\overline{\frac{\mathrm{i} k}{2} \sum_{r+p=k} \hat{\tilde{u}}_{r} \hat{\tilde{u}}_{p}}\right)
$$

is the transfer term due to the nonlinear relation among subscales. All previous relations are defined for $k, q, l \in[-N / 2, N / 2-1], r, p \in[-N, N-2] \backslash[-N / 2, N / 2-1]$, and $t>0$.

\section{Remark 5}

The Galerkin nonlinear term (18) is responsible for the interaction among the resolved scales. The triadic interaction of this term expresses all the possible combinations with the pairs of resolved wavenumbers $q$ and $l$ such that $q+l=k$. In Fig. 1 a brief schematic depicts the triadic interactions between the resolved wavenumbers; the two groups of possible combinations $q+l=k$ include: both $q$ and $l$ smaller than $k$ (at the LHS of the figure), and either one of $q$ or $l$ greater than $k$, added with the remainder conjugate less than $k$ (at the RHS of the figure). The possible combination depicted at the LHS of the figure relates two big resolved scales with one smaller resolved scale. Instead, the combination at the RHS indicates the relation between a resolved scale with a bigger and a smaller resolved scale. It can be proved (like in [21]), that for a given time, $T(k) \in \mathbb{R}$, and that the total energy transfer due to the Galerkin nonlinear term is conserved, i.e., $\sum_{k} T(k)=0$. Therefore, the nonlinear transfer term due to the resolved scales (28), and its respective sign, determines the way the energy is transferred between the resolved scales, in which not only large resolved wavenumbers can transfer energy forward to small resolved wavenumbers, but also the possibility of transferring backwards the energy from small to large resolved wavenumbers exists, as commented before.

Having remarked the importance of the Galerkin nonlinear term, which rules the development of an energy cascade as it transfers energy between the resolved wavenumbers in the inertial range [21], we focus our attention to the terms arising from relations (20), (22), (23) and (24). Such terms represent the interaction between the resolved scales and the subscales, and correspond to the socalled Leonard, Cross, and Reynolds stresses in the standard large eddy simulation (LES) approach to solve turbulent flows. It is worthy to note that the presence of these terms is related only to the introduction of the VMS method, without the modification of the continuous problem that occurs with the LES method.

We can detail the effect of the Cross nonlinear term (22) and (23) by looking to Fig. 2. This figure shows how the subscales play a fundamental role in the resolved scales equation by means of this term. More precisely, the LHS of the figure depicts the possible combinations $q+r=k$ between the resolved and subgrid scales. These type of combinations increase with the resolved wavenumber $k$, and therefore, term (29) can be seen as increasingly responsible for dissipating energy at high wavenumber resolved scales. This will be verified later in the numerical example. On the contrary, for the Reynolds stress term (24) possible combinations $p+r=k$ decrease with the resolved wavenumber. A depiction of that type of combinations involving the subscales is shown in the right side of Fig 2. Therefore, the transfer term due to the nonlinear relation among subscales (30) is essentially related to the dissipation of energy at small resolved wavenumbers.

As in (27), the discrete energy equation for the subscales can be obtained by taking the product of Eq. (20) with the complex conjugate of the subscale $\overline{\left(\hat{\tilde{u}}_{r}\right)}$. If $e_{r}$ is the energy of the subscales, its 

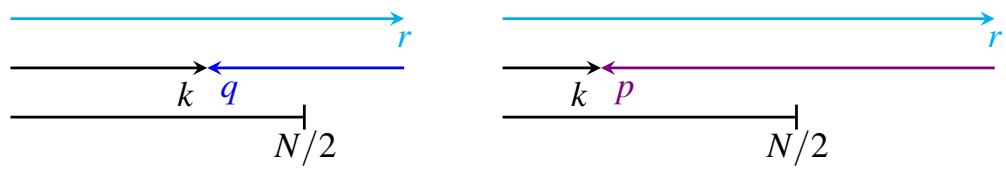

Figure 2. Triadic interactions among the resolved and subgrid scales: Cross stress (left), and Reynolds stress (right).

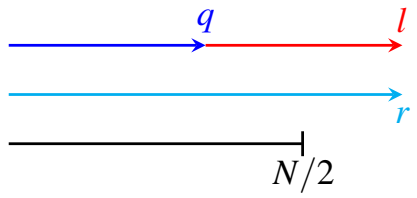

Figure 3. Triadic interactions among the resolved and subgrid scales for the Leonard stress.

time derivative is:

$$
\partial_{t} e_{r}=-2 \tau^{-1}\left(u_{N}\right) e_{r}+L(r),
$$

for all $r \in[-N, N-2] \backslash[-N / 2, N / 2-1]$, and $t>0$. Here we have arranged the nonlinear transfer term due to the residual as:

$$
L(r)=\overline{\overline{\tilde{u}}_{r}}\left(\sum_{q+l=r} \mathrm{i} l \hat{u}_{q} \hat{u}_{l}\right)+\hat{\tilde{u}}_{r}\left(\overline{\sum_{q+l=r} \mathrm{i} l \hat{u}_{q} \hat{u}_{l}}\right),
$$

for all $q, l \in[-N / 2, N / 2-1], r \in[-N, N-2] \backslash[-N / 2, N / 2-1]$, and $t>0$. This transfer term is related to the Leonard stress term and provides the energy that is dissipated by the subscales. An example of the possible combinations between the resolved wavenumbers in the Leonard stress term is presented in Fig. 3, where it can be observed that the possible combinations with the pairs of resolved wavenumbers $q$ and $l$, such that $q+l$, decreases with the subscale wavenumber. As a consequence of this, the rate of energy transfer (and dissipation) of the subscales is in principle smaller for unresolved high wavenumbers.

\section{Remark 6}

The energy transfer terms (29), (30), and (32) allow for the backward transfer of energy from the subscales to the resolved scales, provided the sign of those terms is negative for certain resolved and subscale wavenumbers.

\subsection{Time integration method}

Let $0=t^{0}<t^{1}<\ldots<t^{N}$ be a partition of the time interval of analysis, with $0<\delta t=t^{n+1}-t^{n}$ for $n=0,1,2, \ldots$. Here and below the superscript denotes the time step counter. The transient term integration of the evolution equation for the resolvable scales and the subscales, as well as the energy equations, can be described as follows. Let us write these equations in the form:

$$
\partial_{t} v_{k}=\mathscr{F}\left(v_{k}\right),
$$

for each $k$ wavenumber. We use a fully explicit Runge-Kutta time-integration scheme of $S$ stages. If $v_{k}^{n}$ is known, the solution at $t^{n+1}$ is given by

$$
v_{k}^{n+1}=v_{k}^{n}+\delta t \sum_{i=1}^{S} \mathrm{~b}_{i} K_{i}^{n},
$$


for each $k$ wavenumber, where $K_{i}^{n}$ (defined for $\left.1 \leq i \leq S\right)$ are the stage increments, obtained as

$$
K_{i}^{n}=\mathscr{F}\left(v_{k}^{n}+\delta t \sum_{j=1}^{i-1} \mathrm{a}_{i j} K_{j}^{n}\right) .
$$

Expresions (34) and (35) depend on the definition of the coefficients $\mathrm{a}_{i j}$ (for $1 \leq j \leq i \leq S$ ) and $\mathrm{b}_{i}$ (for $1 \leq i \leq S$ ) for a specific explicit method. In particular, the explicit Euler scheme corresponds to $S=1$ and $b_{1}=1$. A stability condition of Courant-Friedrichs-Lewy type must be imposed in order to guarantee stability of the time integration method. The previous time marching technique applied to Eqs. (25), (20), and (27), defines the spatial and temporal OSGS-VMS discretization of the Burgers problem. In the following section we apply this discretized formulation in order to simulate a numerical example.

\section{NUMERICAL EXAMPLE}

In this section we solve a numerical example that is selected to test the OSGS-VMS formulation. We first define the continuous problem, which is an steady one-dimensional viscid case. The fact that the "turbulent" energy spectrum of the one-dimensional Burgers problem is only due to the resolution of strong gradients in the solution (shocks), and not to the unsteady solution of the problem (as in the case of the dissipative eddies in the Navier-Stokes turbulence), has prompted us to select the problem as a steady case. Then we present the DNS of the problem, which accounts for all the wavenumbers in the solution. The DNS solution is used to evaluate the accuracy of the OSGS-VMS method. Specifically, we discuss the resulting energy spectra for discretizations in which the number of modes $N$ is not enough to resolve all of the wavenumbers present in the solution. As in (21), we can consider an effective grid size $h=\pi / N$ and refer to coarse and fine grids. In the spirit of ILES methods, coarse grid solutions are also compared against LES results in the last part of this section.

Under certain conditions (detailed in [30]), the periodic solution of the one-dimensional forced Burgers problem tends toward a stationary state. This is achieved in particular by selecting $\hat{u}_{0}^{0}=0$ and $\hat{u}_{k}^{0}=k^{-1}$ as initial conditions, and by fixing $\partial_{t} \hat{u}_{1}=0$ for $t>0$. Given these conditions, the diffusivity coefficient of the example is fixed to $v=0.025$, so that the nonlinear term is dominant in the Burgers equation. The periodic solution describes a single shock that arises from the largescale restriction over $\hat{u}_{1}$ and the predominance of the nonlinear term. This restriction also provides the amount of energy that is propagated through the simulation and dissipated by the center of the shock. In this sense, the smallest scales corresponding to the "Kolmogorov" scale belong to the viscous shock thickness $l \approx v /|u|$, and the maximum resolved wavenumber of the DNS solution is calculated such that the associated grid spacing is below this size. The DNS solution of the numerical example is carried out by completely solving all the scales, which is achieved with $N=400$ (so that $h=7.85 \times 10^{-3}$ is below $l \approx 10^{-2}$ ). We also perform simulations in which the grid is not fine enough to resolve all the wavenumbers of the solution. The comparison of the coarse grid simulations (solved with a maximum resolved wavenumber $N$ ) and the DNS solution is explained below. In order to guarantee numerical stability, the time integration stability criterion is set for all the resolved cases to a CFL number smaller that 0.01 for the linearized problem, converging to the steady state at approximately $t=2$.

Figure 4 shows the energy spectrum at the stationary converged state for the DNS and coarse grid simulations. The energy spectrum at the initial simulation time has a regular distribution and behaves as $k^{-2}$, with the large scales containing the majority of the energy in the system. As it evolves in time, the energy spectrum develops the cascade shape with the typical slope -2 (in a log scale) behavior in the inertial range (that differs from the well-known $k^{-5 / 3}$ behavior of the Navier-Stokes turbulence), and a drop-off corresponding to the dissipation provided by the shock. The decay in time of the energy spectra solved by the OSGS-VMS method is accurate for all the coarse grids, giving the $k^{-2}$ behavior at the inertial range, with only small deviations occurring near the maximum resolved wavenumber. In this sense, an energy pile up is observed for a few wavenumbers below the maximum resolved wavenumber. It is also observed that refining the grid, 


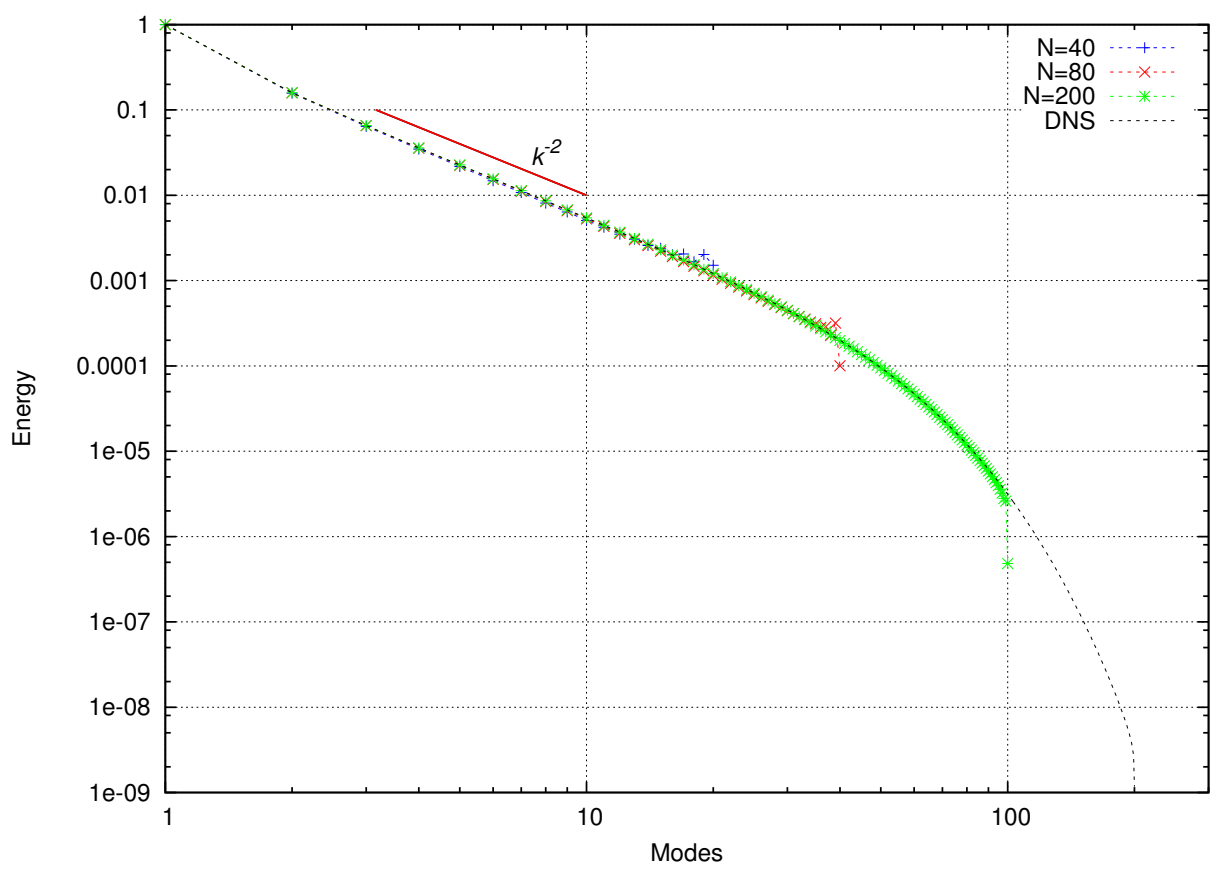

Figure 4. OSGS-VMS results: energy spectrum.

and consequently enriching the space of the subscales, improves the accuracy of the energy spectrum near the maximum resolved wavenumber as the dissipation of energy increases and the pile up vanishes. These results indicate that the turbulent energy spectrum is correctly approximated by the OSGS-VMS numerical method. Even for coarse discretizations that are not sufficient to solve the smallest scales of the solution, the inertial range of the energy spectrum is accurately captured.

Let us discuss now the solution to the Burgers problem in the physical space. Figure 5 presents the physical space solution, including the coarse grid solutions and the DNS solution. The physical space solution $u_{N} \in \mathbb{R}$ is plotted in the range $[0,2 \pi]$, since the solution is periodic. In general, the OSGS-VMS method gives an accurate physical solution, including the periodic wave behavior and the formation of the one-dimensional shock as a step in the solution. For the coarse grid solutions the Gibbs phenomenon is present. This can be observed in the figure as local oscillations near the shock, which are commonly eliminated through shock capturing techniques. A slight inaccuracy different from the Gibbs phenomenon is observed for the coarsest grid solutions, which corresponds to the previously described energy pile up at the highest resolved wavenumbers.

Now we center the discussion about the implicit modeling of turbulence done by the OSGSVMS method. As in the case of the Navier-Stokes turbulence, the forced Burgers problem exhibits the energy dissipation cascade, in which the energy of the large scales is transferred to the small scales where the energy is dissipated. The triadic interaction mechanism commented in Section 3, corresponding to the nonlinear term involving the resolved scales, is responsible for the energy transfer from the preponderant resolved large scales to the much smaller resolved scales. The mechanisms through which the energy contained in the resolved and unresolved scales interact with each other can be clarified by analyzing the scale dependence and the dissipation driven by the nonlinear terms. For doing so, we plot the spectral eddy viscosity arising from the Cross and Reynolds stress terms, which is a scaled version of the energy dissipated by each nonlinear term, and it is calculated as $v_{C}(k)=C(k) / k^{2} e(k)$, and $v_{R}(k)=R(k) / k^{2} e(k)$, where $e(k)$ is the energy of the wavenumber. We plot the scale dependence of the nonlinear terms for a $N=80$ case in Fig 6. It has been previously explained that the Cross stress contribution (involving the resolved and unresolved scales) dissipates energy increasingly with the resolved wavenumber. On the contrary, 


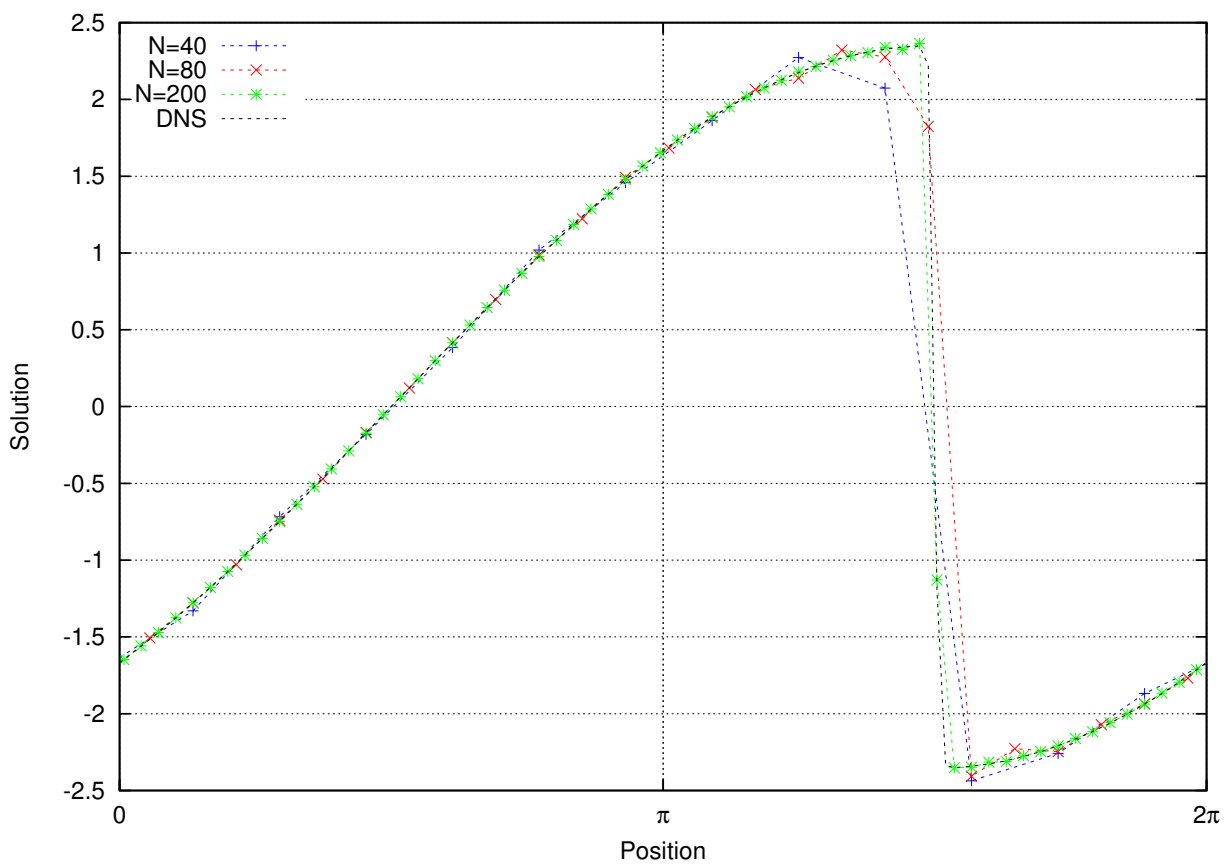

Figure 5. OSGS-VMS results: physical space solution.

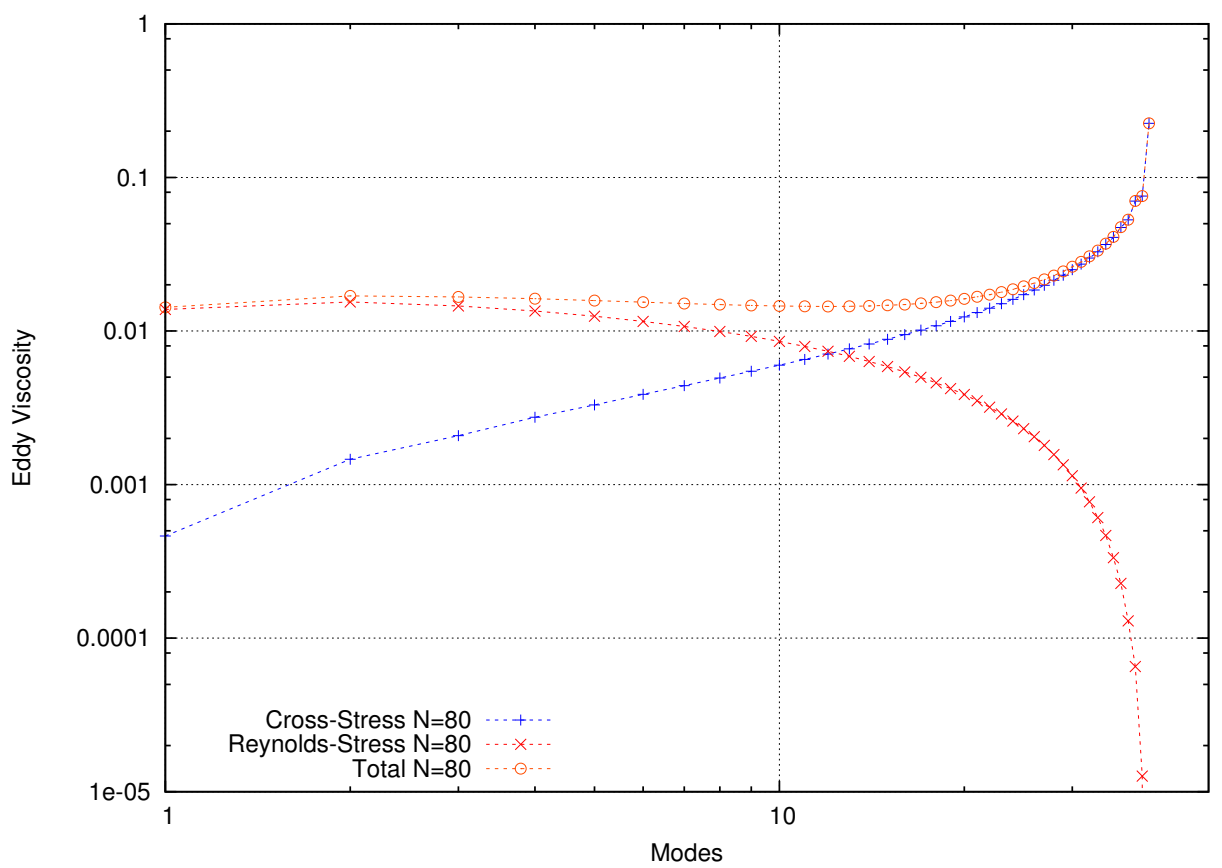

Figure 6. OSGS-VMS results: Eddy viscosity.

the energy dissipation related to the Reynolds stress is greater for the largest scales of the solution. In fact, the largest scales of turbulence are only correctly solved if the nonlinear term accounting for the unresolved scales is included. Contrary to [28], where the stabilizing term was defined by a linearized asymptotic expansion over the residual, and hence only Cross stress terms appeared in 
the discretized equation, we find that including the Reynolds stress tensor (resulting from nonlinear terms involving the subscales) is a requirement for the proper stabilization of the problem, but also for the correct approximation of the turbulence phenomena. In this sense, we also find that including the transient term in the subscales equation (14), and therefore solving the subscales equation as a separate equation, makes the Leonard stress term to be explicitly defined and improves the numerical convergence to the steady state of the solution. Adding the dissipation provided by the Cross and Reynolds terms results in a plateau distribution of the spectral eddy viscosity regardless of the resolved wavenumber. The dissipation increases only at higher resolved wavenumbers by the effect of the Cross stress term. It is remarkable that the numerical dissipation given by the OSGSVMS method removes energy at a correct rate, mainly due to the relation between the variational subscales and the finite resolved scales through the nonlinear terms, which improves by enriching the discretization.

We aim to compare now the previous results with some Burgers turbulence results obtained with a LES model. First, let us briefly comment about the modifications in the continuous Burgers equation when the LES method is applied. The LES method filters the Burgers equation in space and time, leading to the problem

$$
\partial_{t} \underline{u}+\partial_{x}\left(\frac{1}{2} \underline{u u}\right)=v \partial_{x x} \underline{u}+\underline{f}, \quad x \in(0,2 \pi), t>0 .
$$

We have denoted the filtered quantities by using an underline. The filtered nonlinear term $u u$ is the main difficulty in LES: it requires knowledge of the unfiltered velocity field a priori, which is unknown, so it must be modeled somehow. In this sense, the filtered nonlinear term can be split up in the form $\underline{u} \underline{u}=\mathscr{T}+\underline{u} \underline{u}$, where the quantity $\underline{u} \underline{u}$ is resolved. The introduced residual subfilter-scale stress tensor $\mathscr{T}$ must be taken into account in the solution of Eq. (36). This tensor has to be modeled in terms of the filtered unknown in order to close the filtered equation. The static Smagorinsky model $\mathscr{T} \approx-2 v_{t} \mathscr{S}$ introduced in [26] is a common closure model for this problem, where $\mathscr{S}=\frac{1}{2} \partial_{x} \underline{u}$ is the one-dimensional strain tensor and $v_{t}$ an additional eddy viscosity. Other closures, such as the Dynamic Smagorinsky, Scale-similarity, Mixed, and Linear unified RANS-LES models, are discussed in [22]. The closure model can be replaced directly in the filtered equation, so that the modified Burgers equation is obtained:

$$
\partial_{t} \underline{u}+\underline{u} \partial_{x} \underline{u}=v_{\mathrm{eff}} \partial_{x x} \underline{u}+\underline{f}, \quad x \in(0,2 \pi), t>0 .
$$

In practice, the main change with respect to the original equation is to modify $v$ by $v_{\text {eff }}=v+v_{t}$. In order to numerically approximate the previous equation, the Fourier-Galerkin method can applied to the previous equation. Using the same notation as before, the discretized problem is then:

$$
\partial_{t} \underline{\hat{u}}_{k}+\frac{\mathrm{i} k}{2} \sum_{k=q+l} \hat{\underline{u}}_{q} \hat{\underline{u}}_{l}+v_{\mathrm{eff}} k^{2} \underline{\hat{u}}_{k}=\hat{f}_{k}
$$

for all $k, q, l \in[-N / 2, N / 2-1]$, and $t>0$. We apply also the time marching scheme described in Section 3 . The eddy viscosity defined in the Fourier space is modeled using a spectral eddy viscosity proposed in [25], in our case taking the cutoff wavenumber as the number of modes, $N$. It is given for each wavenumber $k$ by

$$
v_{t}(k)=v_{t}^{+\infty}\left(\frac{E_{N}}{N}\right)^{1 / 2} v_{t}^{*}\left(\frac{k}{N}\right)
$$

with

$$
v_{t}^{+\infty}=0.31 \frac{5-m}{m+1} \sqrt{3-m} C_{k}^{-3 / 2},
$$

where $m$ is the negative slope of the energy spectrum, $E_{N}$ is the total energy spectrum at the cutoff wavenumber, $C_{k}$ is the Kolmogorov constant, and $v_{t}^{*}$ is a non-dimensional eddy-viscosity, given by

$$
v_{t}^{*}(k)=1+34.5 \mathrm{e}^{-3.03(N / k)} .
$$


Since we may consider that the density is $\rho=1$, the total energy spectrum is such that $E=\int_{\Omega}|\hat{\hat{u}}|^{2} d k$, and therefore, its value at the cutoff wavenumber is $E_{N}=e_{N} / N$. Numerical coefficients must be set in order to close the viscosity model, which must be known a priori. In particular, we need to set the Burgers energy spectrum negative slope, which has been previously shown to be $m=2$, and the Kolmogorov constant $C_{k}$, which we set experimentally to 0.1 in order to obtain the most accurate results.

As the filtering procedure selects only a certain number of wavenumbers to be solved by the spectral method, the resolved scales are the filtered scales below the selected cutoff wavenumber $N$. Figure 7 shows several solutions in the physical space when applying the static Smagorinsky model with different grid resolutions. The figure shows a correct description of the physical solution for all simulations. Gibbs phenomenon close to the shock is also observed. In general, the method is accurate and correctly portrays the physical solution of the Burgers problem with the given numerical coefficients. Figure 8 presents the energy spectrum for the simulations using the static Smagorinsky model. Even though the LES model is accurate for most of the resolved scales of turbulence, the energy spectra of the DNS and the LES solutions differ substantially for some resolved wavenumbers belonging to the inertial range. In this sense, the LES spectrum presents some energy pile up near the cutoff wavenumber, which is stronger for finer grids. This is an important difference between the LES and the OSGS-VMS turbulence modeling; while applications with the former have to care for the range of scales that are correctly solved by the grid, applying the OSGS-VMS method correctly describes the turbulence phenomena no matter the grid resolution. Remarkably, refining the grid improves the accuracy of the OSGS-VMS energy spectrum near the maximum resolved wavenumber, contrary to the decrease in the energy dissipation observed in LES. Numerical experiments indicate that increasing the Kolmogorov constant value improves the accuracy of the energy spectrum in the inertial range, but produces a more pronounced energy pile up at the cutoff wavenumber. These under dissipative solutions behave similar to the Fourier-Galerkin method presented in Fig. 9 for a $N=40$ simulation, which gives globally unstable solutions. In that figure we show the energy spectrum results for Galerkin, OSGS-VMS and LES methods. On the contrary, the LES energy spectrum results are not accurate when the Kolmogorov constant is decreased. In this sense, the LES spectrum falls faster than the DNS one, and even some energy pile up near the cutoff wavenumber occurs. The possibility of obtaining an over-dissipative character is well known for the LES models: even though the large scales of turbulence may be correctly solved, the energy spectra of the LES solution can differ substantially from DNS due to the enforcement of the energy dissipation for the smaller resolved scales belonging to the inertial range. Another substantial difference between the OSGS-VMS and LES methods is that, while for the OSGS-VMS the calculation of the energy in (27) and (31) is performed only as a post-process (for illustrating purposes), the discrete energy equation for the resolved scales needs to be solved at each time step for the calculation of the LES spectral eddy viscosity, with the consequent increment in the computational cost.

Finally, we address the scale dependence of the LES and the OSGS-VMS nonlinear terms spectral eddy viscosity by using a grid resolution and cutoff wavenumber of $N=80$. The plot of the eddy viscosity with respect to the resolved wavenumbers is presented in Fig 10. It can be seen that the eddy viscosity given by the static Smagorinsky model is almost flat, only increasing at high resolved wavenumbers. The amount of dissipation given by the LES model is tightly related to the parameters of the spectral model. Decreasing the Kolmogorov constant intensifies viscosity, but also makes the solution over-dissipative (as demonstrated in Fig. 9). In the case of the OSGS-VMS method, even if the obtained eddy viscosity is higher than the one obtained with the LES results, the energy spectrum is more accurate than the one obtained with the $C_{k}=0.05$ LES model. The OSGS-VMS viscosity exhibits a sharp eddy increment near the cutoff wavenumber that is not observed for the LES results. Numerical experiments with finer grids confirm this sharp behavior, which is defined solely by the numerical terms involving the variational subscales, and without the need of tuning numerical parameters.

We can effectively conclude that the contribution from the Galerkin form of the problem is not sufficient to correctly reproduce the turbulent energy spectrum. Moreover, it is clear that for coarse 


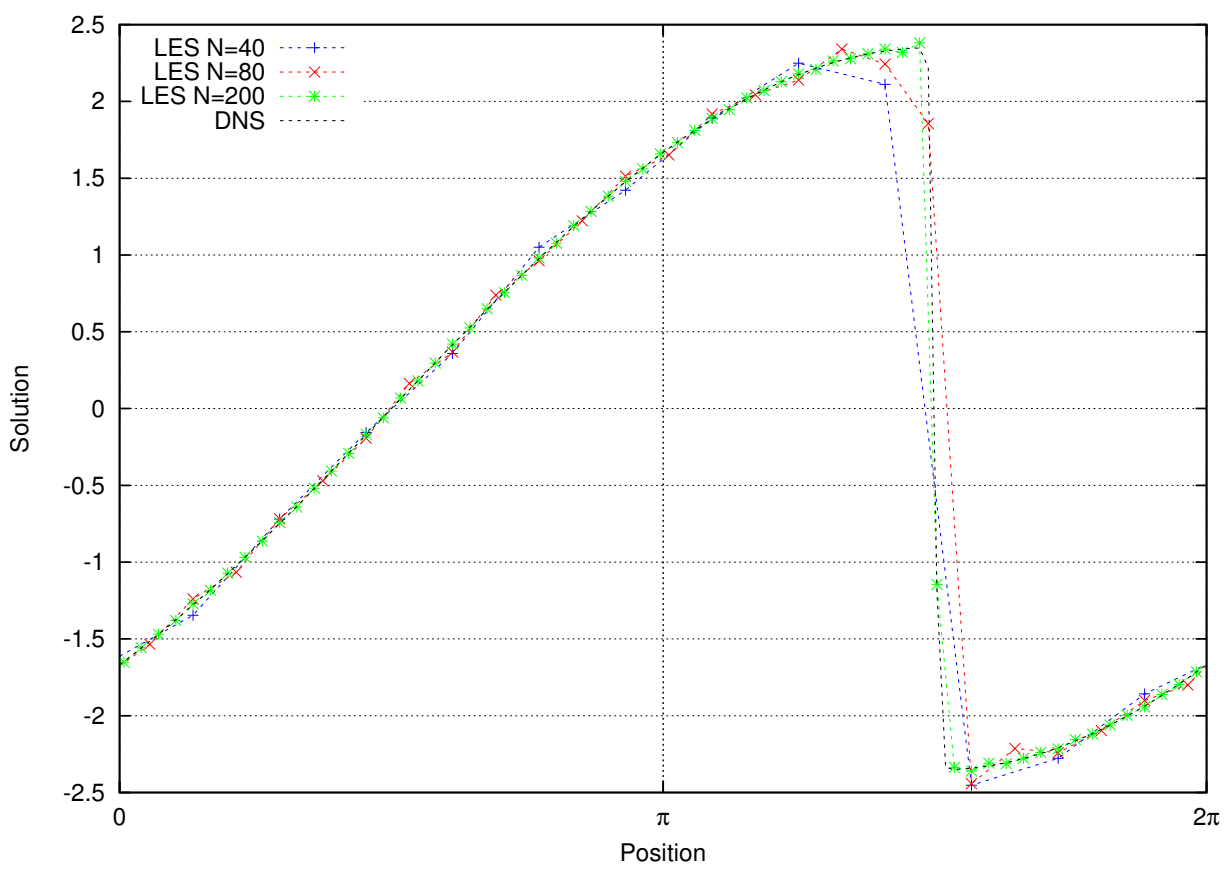

Figure 7. LES results: physical space solution.

discretizations the present OSGS-VMS formulation works as a turbulence model, only depending on the approximations made to derive the subscales (OSGS method and $\tau$ definition). The numerical terms involving the dynamic evolution of the subscales seem to accurately dissipate the energy of the system and to account for the subscale "turbulent effects" onto the resolved scales even better than former LES methods. In the spirit of the discussion in [22], the results of the present work encourage the application of ILES numerical methods with built-in numerical dissipation instead of LES methods. Nevertheless, this conclusion is drawn from the particular case of the steady turbulent solution obtained with the OSGS-VMS method, and the static Smagorinsky model with spectral eddy viscosity.

\section{CONCLUSIONS}

In this work, an Orthogonal Sub-Grid Scales - Variational Multiscale (OSGS-VMS) method for numerically approximating the Burgers problem has been presented. The analysis of the Burgers equation has been done in the Fourier space, which allows us to clarify the scale dependence of the numerical diffusion introduced by the variational multiscale method. In particular, the numerical dissipation introduced with the orthogonal sub-grid scales has been explained in detail. For this, the space for the orthogonal subscales has been defined in terms of the finite-dimensional resolved space, so that the numerical approximation of the unresolved scales improves as the grid is refined. Results have been contrasted with DNS simulations, validating the ability of the OSGS-VMS model to describe the turbulent behavior of the Burgers equation. The scale dependence of the introduced numerical dissipation terms has been also compared with a spectral LES method. An accurate dissipative structure for the OSGS-VMS method has been found, which arises from the numerical approximation exclusively, and without the need of modifying the continuous equation.

As a future work we plan to extend this formulation in order to solve more general problems, specially three dimensional periodic cubes, which we expect to be straightforward. In addition, this work serves as a first approach on the definition of the subscales as both $L^{2}-$ orthogonal and $H^{1}-$ 


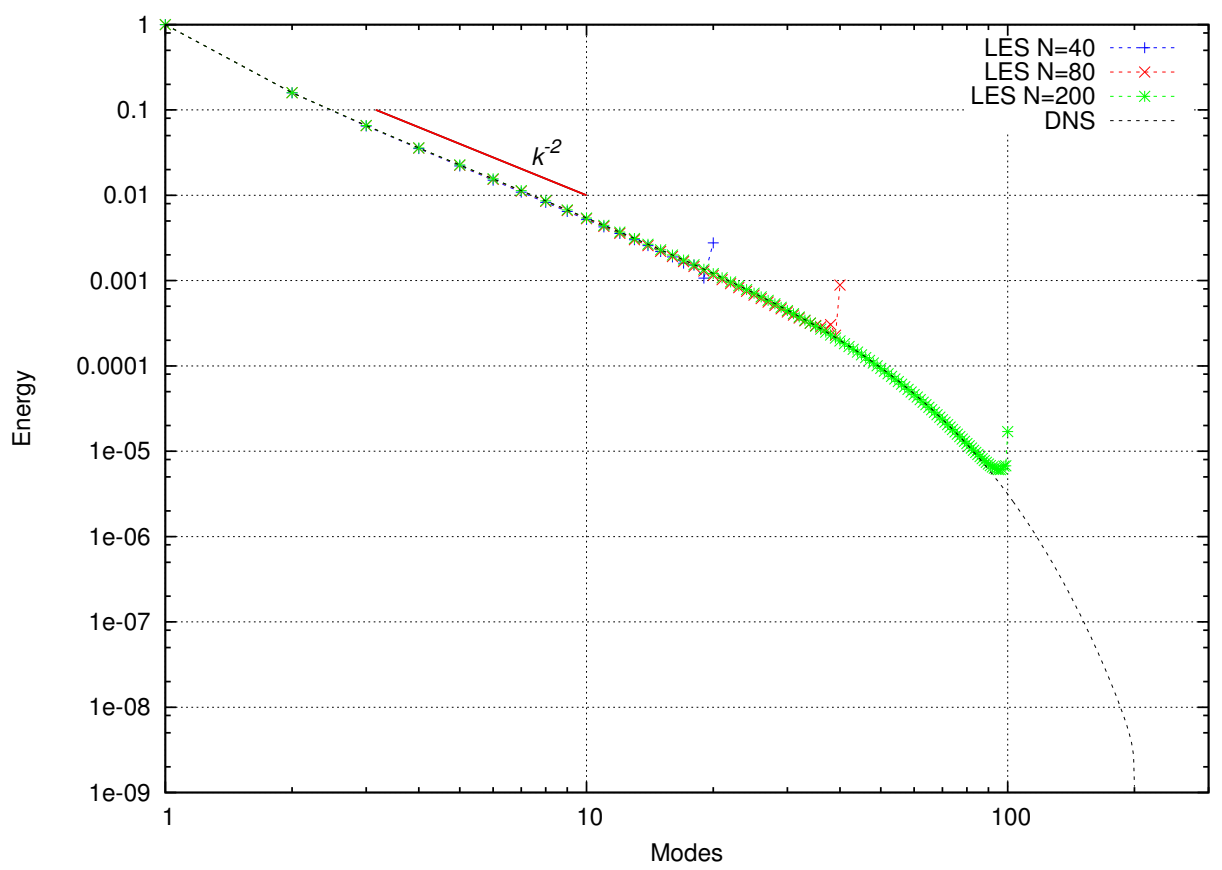

Figure 8. LES results: energy spectrum.

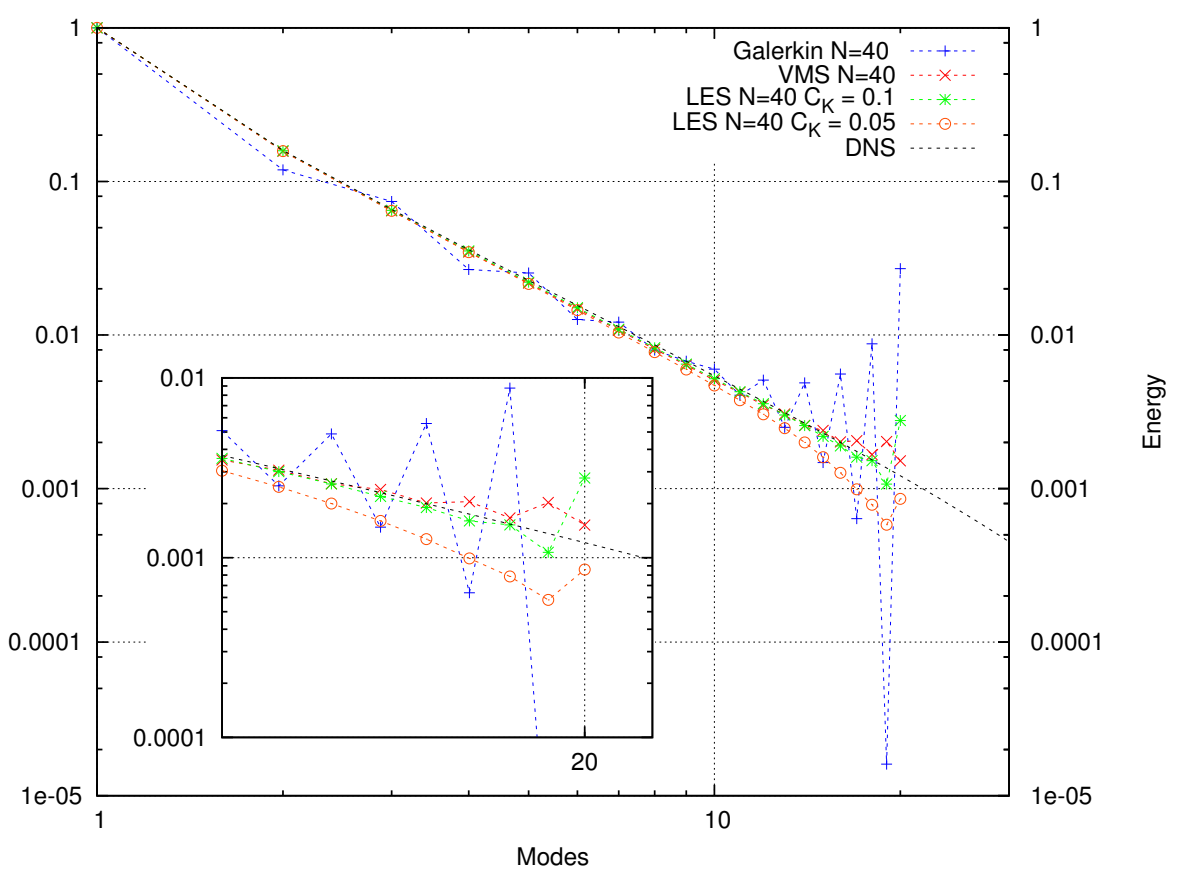

Figure 9. Energy spectrum results for Galerkin, OSGS-VMS, and LES simulations.

orthogonal to the finite element space. Therefore, an expected future work will be to include the $H^{1}$ - orthogonal subscales to the finite element space in several flow problems. For this goal, we first plan to apply this concept to the incompressible Navier-Stokes equations, with the consequence hope that the implicit turbulence modelling will be more accurate. Likewise, the energy budget 


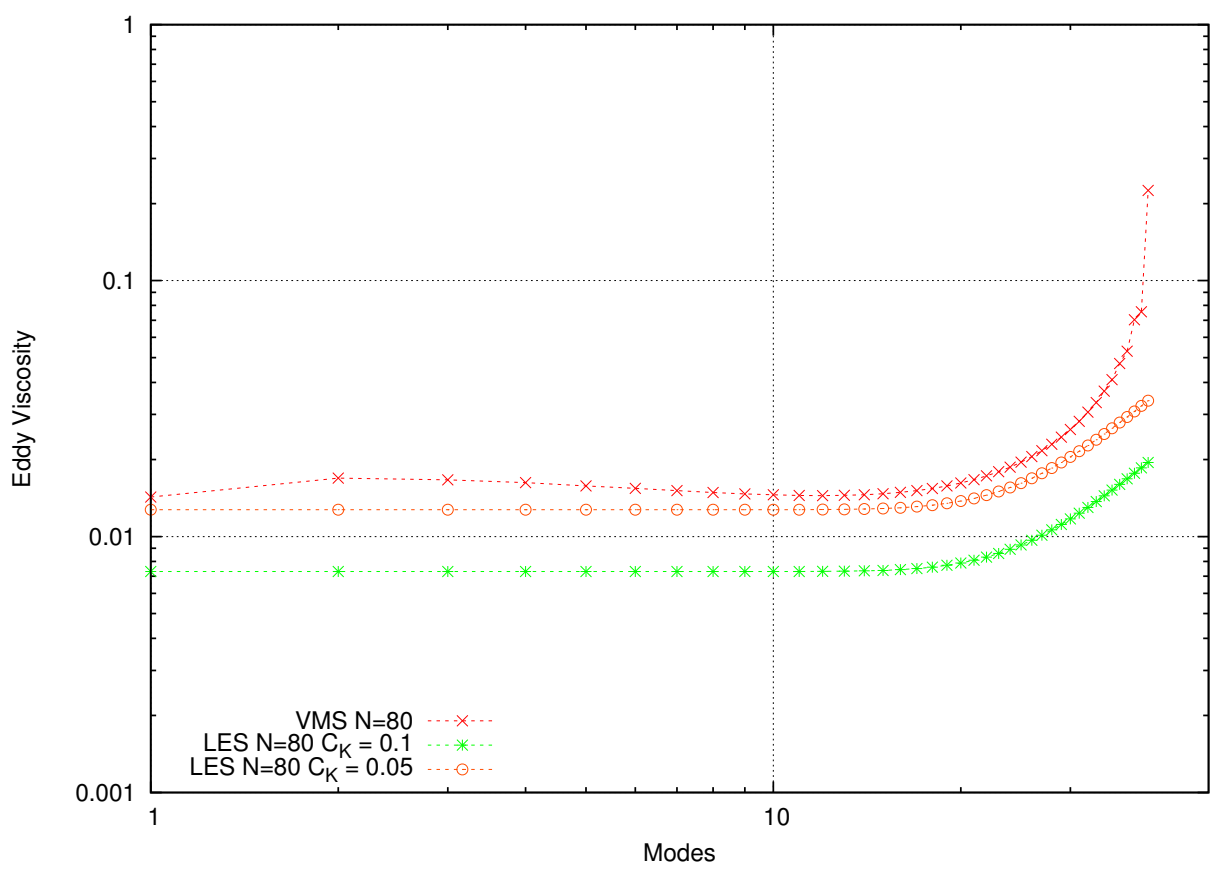

Figure 10. Eddy viscosity results for OSGS-VMS and LES simulations.

between finite element scales and sub-grid scales involves the same transfer of energy from the resolved scales to the sub-grid scales as viceversa (see [7], equations (43) an (44), in the case of the incompressible Navier-Stokes problem).

\section{ACKNOWLEDGEMENTS}

C. Bayona acknowledges the doctoral scholarship received from the Colombian Government-Colciencias. J. Baiges acknowledges the support of the Spanish Government through the Ramón y Cajal grant RYC-201517367. R. Codina acknowledges the support received from the ICREA Acadèmia Program, from the Catalan Government. This work is partially funded through the ELASTIC-FLOW project, Ref. DPI2015-67857-R of the Spanish Government.

\section{REFERENCES}

1. Y. Bazilevs, V. Calo, J. Cottrell, T. J. R. Hughes, A. Reali, and G. Scovazzi. Variational multiscale residual-based turbulence modeling for large eddy simulation of incompressible flows. Comput. Methods. Appl. Mech. \& Eng., 197(1):173-201, 2007

2. J. M. Burgers. The nonlinear diffusion equation. D.Reydel publishing company, Boston, 1974.

3. J. M. Burgers. The nonlinear diffusion equation: asymptotic solutions and statistical problems. Springer Science \& Business Media, 2013.

4. R. Codina. Stabilization of incompressibility and convection through orthogonal sub-scales in finite element methods. Comput. Methods. Appl. Mech. \& Eng., 190(13):1579-1599, 2000.

5. R. Codina. A stabilized finite element method for generalized stationary incompressible flows. Comput. Methods. Appl. Mech. \& Eng., 190(20):2681-2706, 2001.

6. R. Codina. Stabilized finite element approximation of transient incompressible flows using orthogonal subscales. Comput. Methods. Appl. Mech. \& Eng., 191(39):4295-4321, 2002.

7. R. Codina, J. Principe, and M. Ávila. Finite element approximation of turbulent thermally coupled incompressible flows with numerical sub-grid scale modelling. International Journal of Numerical Methods for Heat \& Fluid Flow, 20(5):492-516, 2010.

8. R. Codina, J. Principe, O. Guasch, and S. Badia. Time dependent subscales in the stabilized finite element approximation of incompressible flow problems. Comput. Methods. Appl. Mech. \& Eng., 196(21):2413-2430, 2007. 
9. J. D. Cole. On a quasi-linear parabolic equation occurring in aerodynamics. Quarterly of applied mathematics, 9(3):225-236, 1951.

10. O. Colomés, S. Badia, R. Codina, and J. Principe. Assessment of variational multiscale models for the large eddy simulation of turbulent incompressible flows. Comput. Methods. Appl. Mech. \& Eng., 285:32-63, 2015.

11. A. Das and R. D. Moser. Optimal large-eddy simulation of forced Burgers equation. Physics of Fluids, 14(12):4344-4351, 2002.

12. D. Forti and L. Dedè. Semi-implicit BDF time discretization of the Navier-Stokes equations with VMS-LES modeling in a high performance computing framework. Computers \& Fluids, 117:168-182, 2015.

13. M. Germano, U. Piomelli, P. Moin, and W. H. Cabot. A dynamic subgrid-scale eddy viscosity model. Physics of Fluids A: Fluid Dynamics (1989-1993), 3(7):1760-1765, 1991.

14. B. J. Geurts and D. D. Holm. Regularization modeling for large-eddy simulation. Physics of Fluids (1994-present), 15(1):L13-L16, 2003.

15. V. Gravemeier and W. A. Wall. An algebraic variational multiscale-multigrid method for large-eddy simulation of turbulent variable-density flow at low mach number. Journal of Computational Physics, 229(17):6047-6070, 2010.

16. O. Guasch and R. Codina. Statistical behavior of the orthogonal subgrid scale stabilization terms in the finite element large eddy simulation of turbulent flows. Comput. Methods. Appl. Mech. \& Eng., 261:154-166, 2013.

17. J. Hoffman and C. Johnson. A new approach to computational turbulence modeling. Comput. Methods. Appl. Mech. \& Eng., 195(23):2865-2880, 2006.

18. E. Hopf. The partial differential equation $u_{t}+(u u)_{x}=\mu u_{x x}$. Communications on Pure and Applied Mathematics, 3(3):201-230, 1950.

19. T. J. R. Hughes. Multiscale phenomena: Green's functions, the Dirichlet-to-Neumann formulation, subgrid scale models, bubbles and the origins of stabilized methods. Comput. Methods. Appl. Mech. \& Eng., 127(1):387-401, 1995.

20. T. J. R. Hughes, V. M. Calo, and G. Scovazzi. Variational and multiscale methods in turbulence. In Mechanics of the 21st Century, pages 153-163. Springer, 2005.

21. A. K. Kuczaj, B. J. Geurts, and W. D. McComb. Nonlocal modulation of the energy cascade in broadband-forced turbulence. Physical Review E, 74(1):016306, 2006.

22. Y. Li and Z. Wang. A priori and a posteriori evaluations of sub-grid scale models for the Burgers' equation Computers \& Fluids, 139:92-104, 2016.

23. J. Liu and A. Oberai. The residual-based variational multiscale formulation for the large eddy simulation of compressible flows. Comput. Methods. Appl. Mech. \& Eng., 245:176-193, 2012.

24. P. J. Mason and D. Thomson. Stochastic backscatter in large-eddy simulations of boundary layers. Journal of Fluid Mechanics, 242:51-78, 1992.

25. O. Métais and M. Lesieur. Spectral large-eddy simulation of isotropic and stably stratified turbulence. Journal of Fluid Mechanics, 239:157-194, 1992.

26. J. Smagorinsky. General circulation experiments with the primitive equations: I. The basic experiment. Monthly weather review, 91(3):99-164, 1963.

27. K. S. Surana, S. Allu, J. Reddy, and P. Tenpas. Least-squares finite element processes in h, p, k mathematical and computational framework for a non-linear conservation law. International Journal for Numerical Methods in Fluids, 57(10):1545-1568, 2008.

28. Z. Wang and A. Oberai. Spectral analysis of the dissipation of the residual-based variational multiscale method. Comput. Methods. Appl. Mech. \& Eng., 199(13):810-818, 2010.

29. C. E. Wasberg, T. Gjesdal, B. A. P. Reif, and Ø. Andreassen. Variational multiscale turbulence modelling in a high order spectral element method. Journal of Computational Physics, 228(19):7333-7356, 2009.

30. G. Wei and Y. Gu. Conjugate filter approach for solving Burgers equation. Journal of Computational and Applied Mathematics, 149(2):439-456, 2002. 